\title{
Dipping and Absorption in the stellar wind in GX 301-2
}

\section{Ingo Kreykenbohm*}

Dr. Karl Remeis-Observatory \& ECAP, Universität Erlangen-Nürnberg, Sternwartstr. 7, 96049

Bamberg, Germany

E-mail: ingo.kreykenbohmesternwarte.uni-erlangen.de

\section{Ersin Göğüş}

Sabancl University, Faculty of Engineering and Natural Sciences, Orhanl- Tuzla, 34956

Istanbul, Turkey

E-mail: ersingesabanciuniv.edu

\section{Tomaso M. Belloni}

INAF-Osservatorio Astronomico di Brera, Via E. Bianchi 46, I-23807 Merate (LC), Italy

E-mail: tomaso.belloni@brera.inaf.it

\section{Felix Fürst, Jörn Wilms}

Dr. Karl Remeis-Observatory \& ECAP, Universität Erlangen-Nürnberg, Sternwartstr. 7, 96049 Bamberg, Germany

E-mail: felix.fuerstesternwarte.uni-erlangen.de, joern.wilmsesternwarte.uni-erlangen.de

\section{Slawomir Suchy, Richard E. Rothschild}

University of California, San Diego, Center for Astrophysics and Space Sciences, 9500 Gilman Drive, La Jolla, CA 92093-0424

E-mail: ssuchy@ucsd.edu,richardr@ucsd.edu

We present a detailed study of the absorption and dipping behavior of the accreting High Mass Xray Binary GX 301-2. We use data from the XMM-Newton observatory to study the low energy continuum and the iron line complex and data from the Rossi X-ray Timing Explorer (RXTE) to model the continuum and study lightcurves. The analysis of the RXTE data shows that the source is strongly variable: the source exhibits intense flaring as well as dipping activity. The source countrate as recorded by the RXTE-PCA drops to almost zero during some of the dips in the lightcurve. A similar dipping behavior has been observed in the Vela X-1 as well as a number of other massive wind accreting sources. We present a detailed spectral and temporal study of the dips and discuss possible scenarios to explain the dips.

The high quality XMM timing mode data are taken during the pre-periastron flare. We accumulate an overall spectrum with very high signal to noise ratio and model it with a partial covering model. The high signal allows us to obtain spectra as short as $30 \mathrm{~s}$ to track the evolution of the photoelectric absorption and thus the density of the material in the stellar wind.

8th INTEGRAL Workshop "The Restless Gamma-ray Universe"

September 27-30 2010

Dublin Castle, Dublin, Ireland

\footnotetext{
* Speaker.
} 


\section{Introduction}

GX 301-2 (also known as 4U 1123-62) is a High Mass X-ray Binary (HMXB) consisting of a neutron star and the early type companion BP Cru. The neutron star is in an eccentric orbit (e $=0.462$ ) with an orbital period of about 41.5 days [4]. The companion star is was first classified as a B1.2Ia star [4], while it was later reclassified as B1 Iae+ hypergiant [3]. This more recent classification makes Wray 977 with a luminosity of $1.3 \times 10^{6} L_{\odot}$ and a mass of at least $48 M_{\odot}$ one of the most luminous stars in our galaxy. The star has a very high mass loss rate of $\dot{M} \sim 10^{-5} M_{\odot} \mathrm{yr}^{-1}$ resulting in a very dense but slow $\left(v_{\infty}=400 \mathrm{~km} \mathrm{~s}^{-1}\right)$ stellar wind.

The neutron star rotates with a period of approximately $686 \mathrm{~s}$ [14]. It accretes material directly from the dense stellar wind, resulting in a highly variable X-ray light curve, typical for wind accretion. X-ray luminosity changes by a factor of five or more within one hour are common in GX 301-2 [12]. The orbital light curve exhibits significant variability: shortly before periastron, the neutron star encounters a gas stream flowing from the optical companion to the neutron star resulting in intense flaring activity [11]. Following the periastron, the X-ray intensity reaches a minimum around orbital phase 0.2 as the neutron star passes through the dense inner regions of the stellar wind until it is almost eclipsed by the stellar companion [8]. The X-ray intensity increases again towards a weak secondary maximum near apastron.

The X-ray spectrum of GX 301-2 was usually described by a powerlaw modified at higher energies by an exponential cutoff. More recent studies, however, used a partial covering model as the spectrum of GX 301-2 is heavily absorbed [7, 6] with $N_{\mathrm{H}}$ ranging from $10^{23}$ up to $2 \times$ $10^{24} \mathrm{~cm}^{-2}$ [7]. Around $6.4 \mathrm{keV}$ a very strong iron fluorescence line with an equivalent width of up to $1800 \mathrm{eV}$ is present [9]. At energies above $20 \mathrm{keV}$, the spectrum is further modified by the presence of a strongly pulse phase dependent cyclotron resonant scattering feature around $\sim 37 \mathrm{keV}$ $[10,6]$.

\section{Observations}

We have obtained $\sim 50 \mathrm{ksec}$ of high quality data with XMM-Newton on July 12, 2009 (MJD 55024.1037 to MJD 55024.6430). Since the source is extremely bright, the EPIC-PN camera of XMM-Newton was operated in the timing mode and the two EPIC-MOS cameras were switched off and the telemetry assigned to the EPIC-PN camera. The observations were performed during the pre-periastron flare around orbital phase 0.9 where the source is very bright and shows extreme variability and intense flaring activity.

In addition we analyzed $185 \mathrm{ksec}$ of public Rossi X-ray Timing Explorer (RXTE) observations of GX 301-2 which were performed between 2010 May 26 and July 22. Several observations were unusally long: at least $10 \mathrm{ksec}$ of uninterrupted data taking. We used data from all PCUs for timing studies while we used data collected with PCU2 only for time resolved spectral investigations to avoid calibration uncertainties. 


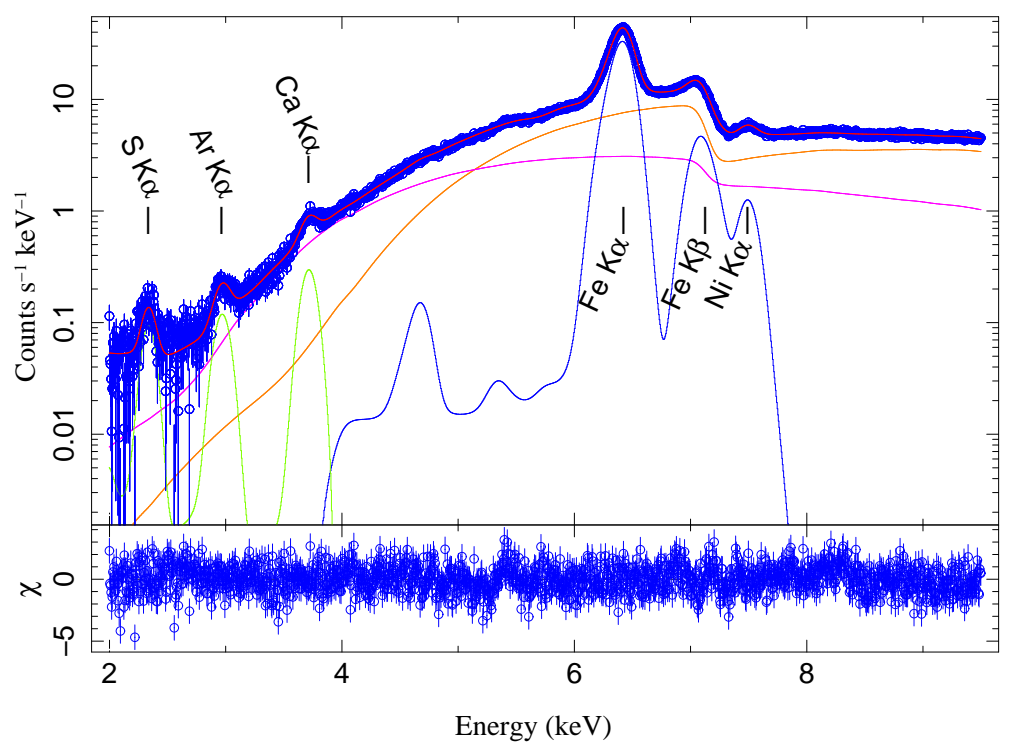

Figure 1: Spectrum of GX 301-2 taken by XMM-Newton. The spectrum was modelled with a partial covering model. Note that the photoelectic absorption is so strong that almost no flux is present below $2 \mathrm{keV}$. The column density results furthermore in very strong iron and nickel fluorescence lines.

\section{Data analysis}

\subsection{XMM-Newton}

We used the complete XMM-Newton observation to obtain a spectrum with the best signal to noise ratio (see Fig. 1). The shape of the continuum is very complex and can not be fitted with simple models such as cutoffpl from XSPEC. We used a partial covering model with both components being strongly absorbed $\left(N_{\mathrm{H}, 1} \sim 130 \times 10^{22} \mathrm{~cm}^{-2}\right.$ and $\left.N_{\mathrm{H}, 2} \sim 50 \times 10^{22} \mathrm{~cm}^{-2}\right)$ plus four Gaussians to model the iron line complex: a neutral iron $\mathrm{K}_{\alpha}$ line at $6.4 \mathrm{keV}$ plus a Compton shoulder, iron $\mathrm{K}_{\beta}$ line at $7.1 \mathrm{keV}$, and Nickel $\mathrm{K}_{\alpha}$ at $7.5 \mathrm{keV}$ (the individual components are shown in Fig. 1). The excellent statistics allow us to obtain spectra with an exposure time of only $30 \mathrm{~s}$. Keeping most continuum parameters fixed, we obtain the $N_{\mathrm{H}}$ for each individual spectrum, i.e. an $N_{\mathrm{H}}$ lightcurve which shows the strong variability of the $N_{\mathrm{H}}$.

\subsection{RXTE}

The analysis of the RXTE/PCA lightcurve reveals a highly unusual behavior on May 28, 2010 [2]: while the source was strongly pulsating with a period of $\sim 686 \mathrm{~s}$, the X-ray intensity was decreasing smoothly, until a flux level of only $\sim 10 \%$ of the original level was reached. This dip itself was about $1000 \mathrm{~s}$ (from MJD 55344.044 to 55344.055) long. During this dip, the pulsations could not be detected (see Fig. 2). After the dip, the X-ray intensity of the source gradually increased again and immediately pulsations resumed. We analyzed all other pointings of GX 301-2, but found no other dipping episode. 


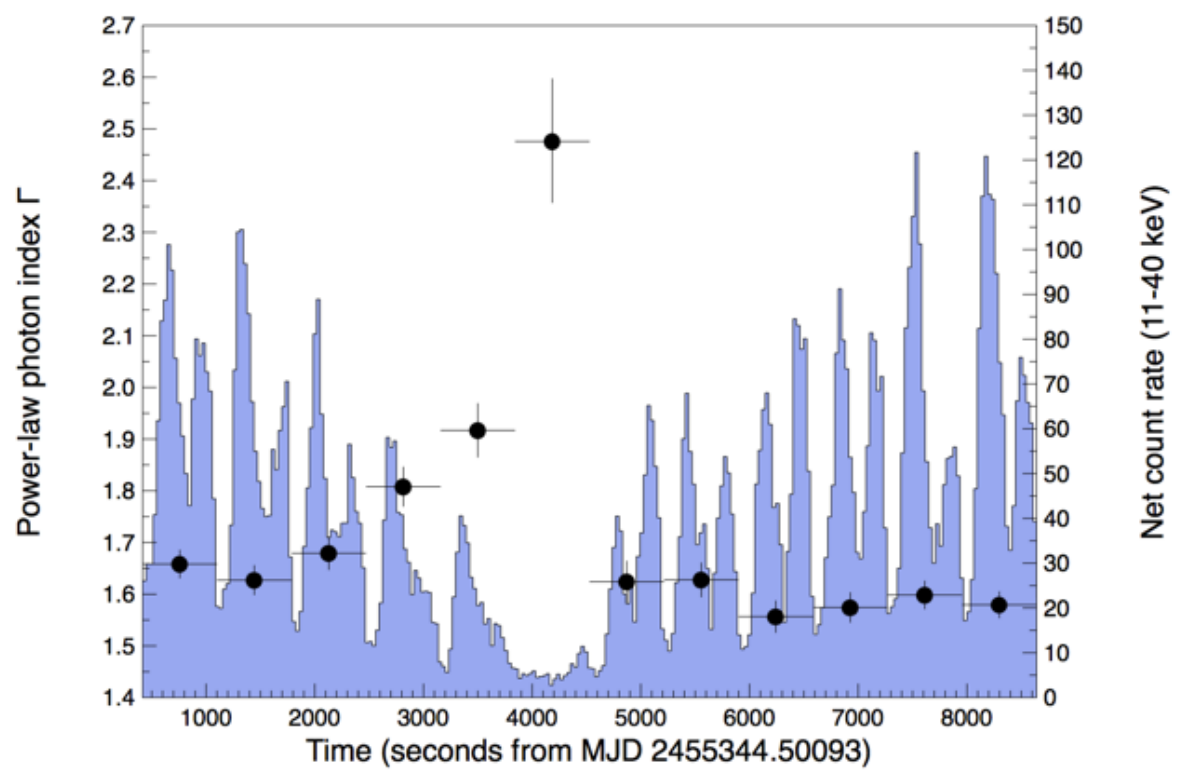

Figure 2: Light curve of RXTE/PCA observations in the 11-40 keV range of GX 301-2 on 2010 May 28 (histogram). A spectrum for each single pulse was extracted and the circles indicate the value of the powerlaw index $\Gamma$ for the individual spectra.

The pulse profile of GX 301-2 exhibits two pulses: a main pulse and a slightly weaker secondary pulse. Up to the dip, the secondary peak is as usual weaker than the main pulse and furthermore the secondary peak decays faster towards the dip than the main peak. During the dip, no significant pulse profile is visible. After the dip, both peaks are abruptly present again, however, the secondary peak is now stronger than the main peak for the next four pulses. After that, the pulse shape of the source resembles its typical long term appearance again.

To further investigate this dip, we performed a time resolved spectral analysis to study the nature of the X-ray emission from GX 301-2 prior to, during, and after the dip. We accumulated pulse phase integrated source and background spectra for the 12 consecutive pulses prior to, during, and following the dip. We modeled the X-ray spectra in the 3-30 keV band, fitting first each spectrum individually with an absorbed power law plus a Gaussian at $6.4 \mathrm{keV}$ to account for the iron fluorescence line. Although this model is rather simple, it describes the data sufficiently well $\left(0.6<\chi_{v}^{2}<1.3\right)$. The hydrogen column density is consistent with a constant value within uncertainties: it varies between $1.3 \times 10^{23}$ and $1.7 \times 10^{23} \mathrm{~cm}^{-2}$. In the next step, we therefore performed a simultaneous fit of all 12 spectra together, using the same absorption column density, iron line energy and width and leaving only the power law index and the two normalizations free. The resulting best fit yields $\mathrm{N}_{\mathrm{H}}=(1.6 \pm 0.2) \times 10^{23} \mathrm{~cm}^{-2}$ with a $\chi_{\text {red }}^{2}=0.96$. Most interestingly, however, we observe a significant softening of the spectrum of the source already prior and especially during the dip (see Fig. 2). After the dip, the spectrum immediately resumes its pre-dip shape. A more detailed discussion is given in [2]. 


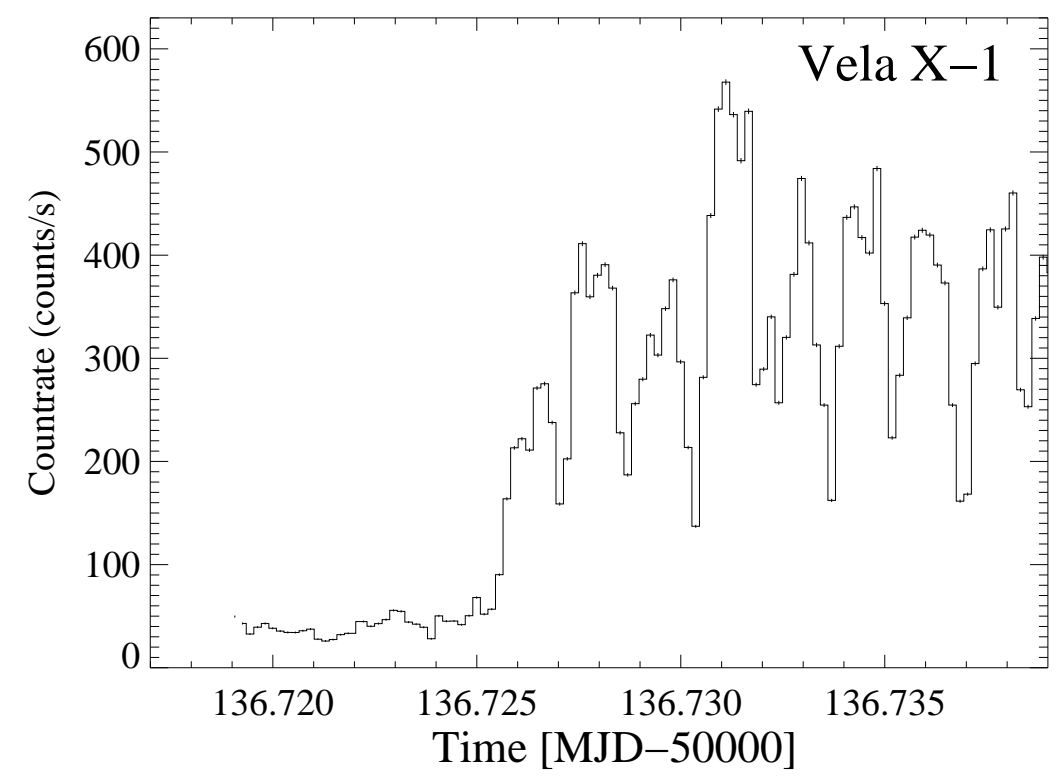

Figure 3: Lightcurve of an observation RXTE observation of Vela X-1 from February 23, 1996. At the beginning of the observation also no pulsations are visible and the overall flux level is very low. After the dip (or off-state) Vela X-1 resumes the normal pulsating behavior.

\section{Discussion}

Although the observed dip in GX 301-2 is rather unique, similar dips or offstates have been observed in other massive wind accreting X-ray binaries.

A very interesting dip was observed in a short RXTE observation of Vela X-1 in 1996 [5]: our re-analysis of these data shows no pulsations are present at the beginning of the observation and the flux was at a very low level ( $\sim 37$ counts/s/PCU in the 3-25 keV band compared to $\sim 300$ counts/s/PCU during the normal bright state; see Fig. 3). We extracted X-ray spectra from the dim non-pulsating state and from the bright pulsating state later in the same observation (see Fig. 3). The spectrum of Vela X-1 is significantly softer during the dim non-pulsating state compared to the normal state. Therefore, GX 301-2 and Vela X-1 show the same behavior: they both exhibit short periods of time during which they are very dim and no pulsations are seen.

The gradual reduction and disappearance of the X-ray pulsations in GX 301-suggests that the $\mathrm{X}$-ray emitting regions of the source were blocked by a dense blob in the stellar wind of the companion. However, the spectrum during such a dip caused by photoelectric absorption is expected to be significantly harder as only the softer X-ray photons are absorbed. Since we observe significant spectral softening during these dips, they can not be caused by simple photoelectric absorption. [1] reported the disappearence of X-ray pulsations in GX $1+4$ during low luminosity states and suggested that they are caused by the propeller effect. Since GX 301-2 is known to have a very high magnetic field strength of the order of $3 \times 10^{12} \mathrm{G}$ [6], it is possible that the infalling material can be expelled via the propeller effect. Unlike the offstates in Vela X-1, the dip in GX 301-2, however, is probably not caused by the propeller effect alone, as the system would be expected to remain in the propeller regime for a longer time than just one pulse period. Another possibility is that there 
could be a short interruption in the accretion stream due to e.g. fluctuations in the stellar wind. In this scenario, the observed low level emission would originate from the surface of the neutron star. During the normal pulsating mode, it would not be possible to disentangle the radiation from the accretion column and the radiation from the surface of the neutron star. Also in this scenario it is not straightforward to explain why the dip lasts exactly one pulse period, however, the time scales predicted for such density fluctuations in the stellar wind match the duration of the dip [13].

\section{Acknowledgments}

This research was funded by the BMWi under DLR grant numbers 50 OR 0801 and 50 OR 0808. Furthermore IK, EG, and TMB acknowledge EU FP6 grant MTKD-CT-2006-042722. TMB acknowledges support from grant ASI-INAF I/009/10/0.

\section{References}

[1] Wei Cui. Evidence for "propeller" effects in X-ray pulsars GX 1+4 and GRO j1744-28. ApJ, 482:L163, Jun 1997.

[2] E. Göğüş, I. Kreykenbohm, and T. M. Belloni. Discovery of a peculiar dip from GX 301-2. A\&A, 525:L6+, January 2011.

[3] L. Kaper, H. J. G. L. M. Lamers, E. Ruymaekers, E. P.J. van den Heuvel, and E. J. Zuiderwijk. Wray 977 (GX 301-2): a hypergiant with pulsar companion. A\&A, 300:446-452, 1995.

[4] Danny T. Koh, Lars Bildsten, Deepto Chakrabarty, Robert W. Nelson, Thomas A. Prince, Brian A. Vaughn, Mark H. Finger, Robert B. Wilson, and Bradley C. Rubin. Rapid spin-up episodes in the wind-fed accreting pulsar GX 301-2. ApJ, 479:933-947, April 201997.

[5] I. Kreykenbohm, P. Kretschmar, J. Wilms, R. Staubert, E. Kendziorra, D. E. Gruber, W. A. Heindl, and R. E. Rothschild. Vela X-1 as seen by RXTE. A\&A, 341:141-150, 1999.

[6] I. Kreykenbohm, J. Wilms, W. Coburn, M. Kuster, R. E. Rothschild, W. A. Heindl, P. Kretschmar, and R. Staubert. The variable cyclotron line in GX 301-2. A\&A, 427:975-986, December 2004.

[7] A. La Barbera, A. Segreto, A. Santangelo, I. Kreykenbohm, and M. Orlandini. A study of an orbital cycle of GX 301-2 observed by BeppoSAX. A\&A, 438:617-632, August 2005.

[8] D. A. Leahy. The rxte/asm X-ray light curve of gx301-2. A\&A, 391:219-224, 2002.

[9] Denis A. Leahy, Masanori Matsuoka, Nobuyuki Kawai, Katsuji Koyama, and Fumiyoshi Makino. Detection of an X-ray intensity dip from GX 301-2. PASJ, 40:197-208, 1988.

[10] T. Mihara. Observational Study of X-ray spectra of binary pulsars with Ginga. PhD thesis, RIKEN, Tokio, June 1995.

[11] Steven H. Pravdo, Charles S. R. Day, Lorella Angelini, B. Alan Harmon, A. Yoshida, and P. Saraswat. ASCA and GROobservations of GX 301-2. ApJ, 454:872-879, December 11995.

[12] Richard E. Rothschild and Yang Soong. Two binary cycles of GX 301-2. ApJ, 315:154-161, April 1 1987.

[13] M. C Runacres and S. P Owocki. A pseudo-planar, periodic-box formalism for modelling the outer evolution of structure in spherically expanding stellar winds. A\&A, 429:323, Jan 2005.

[14] N. Sato, F. Nagase, N. Kawai, R. L. Kelley, S. Rappaport, and N. E. White. Orbital elements of the binary X-ray pulsar GX 301-2. ApJ, 304:241-248, May 11986. 